\section{Les patients victimes d'aléas thérapeutiques (accidents médicaux)}

\author{
Urgence d'établir en Suisse un dispositif d'indemnisation/ \\ compensation
}

J. Martin, Y. Guisan

\section{Une situation difficile qui a retenu l'attention}

En novembre 2001, la Suisse romande a été vivement émue et interpellée par l'histoire personnelle et familiale de Cosette Laurent, dont le grand public a été informé par une émission Temps présent de la Télévision suisse romande du 22 novembre, puis par la grève de la faim que cette personne a entreprise devant l'Hôtel de Ville de Lausanne. Elle a aussi retenu l'attention des médias alémaniques.

En 1989, Mme L. a eu dans un hôpital vaudois une opération gynécologique usuelle, marquée par une complication qui a donné lieu à réintervention ultérieure puis, au cours des années suivantes, à une vingtaine de nouvelles interventions. Aujourd'hui, elle n'urine plus par voie naturelle mais par une sonde sous-ombilicale et présente de sérieuses douleurs chroniques. Elle a été atteinte de manière importante dans son fonctionnement, devant abandonner des activités professionnelles, sportives et sociales, et bénéficie d'une rente AI à 100\%.

S'agissant d'une personne qui avait 35 ans au moment de la première opération (de nature élective), cette séquence d'évènements est dramatique. Suite à la publicité qui lui a été faite, on a vu des milliers de personnes signer une pétition en sa faveur. On a vu de plus affluer les témoignages d'autres «malchanceux de la médecine", souffrant d'effets secondaires et de séquelles douloureuses ou invalidantes liées à des traitements, et qui restent amers, blessés aussi par le fait qu'ils n'ont eu droit à aucune compensation.

Il convient ici de noter que l'enchaînement des circonstances du cas de Mme L. correspond au fonctionnement logique des institutions de notre Etat de droit. Dans un premier temps, une expertise extrajudiciaire avait conclu dans un sens favorable à la patiente, puis un tribunal dans un autre sens. Ainsi,

Correspondance:

Dr Jean Martin, PD

Médecin cantonal

Cité-Devant 11

CH-1014 Lausanne

Dr Yves Guisan

Vice-Président de la FMH

CH-1837 Château d'Oex la notion d'une faute professionnelle qui pouvait ouvrir la voie à un dédommagement financier n'a pas été reconnue. Mme L. s'est alors vue chargée des frais de justice. S'agissant de vicissitudes au terme d'une longue trajectoire de soins qui n'ont pas donné les résultats voulus, c'était beaucoup. On est porté à rappeler l'adage latin summa jus, summa injuria: "le sommet du droit, le sommet de la non-justice». De tels cas surviennent et ils sont toujours regrettables.

\section{La problématique de l'aléa thérapeutique}

Il s'agit donc de ce que nos collègues français appellent aléa thérapeutique ou accident médical: effets délétères de traitements survenus sans qu'il y ait eu faute (ou sans faute démontrée) des prestataires de soins.

$\mathrm{Au}$ cours des quinze dernières années, l'opinion s'est renforcée qu'il n'était pas possible d'en rester à la situation qui est aujourd'hui encore celle de la Suisse, à savoir que, ou bien il y a faute démontrée des prestataires et cela permet une compensation/ indemnisation, ou bien une faute n'est pas prouvée et les patients sont laissés à leur malheur ... Cette évolution de l'opinion est liée à plusieurs facteurs:

- La médecine est devenue de plus en plus efficace dans de nombreux registres et cette efficacité s'accompagne de risques thérapeutiques d'importance. Un médicament ou un moyen donné, s'il a un effet fort à l'égard d'un problème pathologique, peut avoir un effet indésirable marqué à l'endroit de fonctions ou structures physiologiques.

- Quand ces risques sont liés au traitement d'une maladie grave à mauvais pronostic, les praticiens sont sensibilisés au fait de les expliquer adéquatement au patient et le cas échéant à ses proches. Il y a là une sorte de pari: telle affection grave n'est accessible qu'à des moyens «héroïques» et la chance d'une amélioration/guérison est liée à certains dangers; il s'agit de décider en connaissance de cause si on est prêt à encourir ces dangers.

- La situation est à l'évidence différente quand on a à faire à des affections courantes ne menaçant pas la vie, particulièrement quand la question se pose d'intervenir ou à l'inverse de préconiser une abstention thérapeutique et une simple observation. Dans ces cas et de manière compréhensible, des complications sont difficilement acceptées et acceptables, surtout si elles ont des conséquences graves à long terme. Quand de telles atteintes sont survenues sans faute démontrable du prestataire et sont du registre du hasard probabiliste et de la malchance ("la faute à pas de chance»), il devient cependant de plus en plus discutable que les patients restent sans aucune indemnisation (si ce n'est le cas échéant une rente AI). ${ }^{1}$

${ }^{1}$ N.B.: On traite ici des situations sans faute (= responsabilité causale), tout en sachant que, parfois, il subsiste un doute quant à l'absence d'une telle faute. En effet, pas rarement dans notre domaine, il ne s'avère pas possible de rassembler les éléments objectifs permettant une conclusion catégorique. 


\section{Prises de position de la FMH}

La Fédération des médecins suisses s'est penchée à plusieurs reprises sur le problème du risque/accident médical. On relève:

- Un rapport de Me Hans 0tt [1], sur un Congrès britannique et l'éventualité de réviser le régime de la responsabilité médicale dans le sens d'une responsabilité causale (en évoquant les dispositions alors en vigueur en Suède, en Finlande et en Nouvelle-Zélande).

- Une déclaration approuvant la création d'une "Caisse des patients pour les cas de nécessité», là où il n'y a pas eu de faute professionnelle et où les prestations des assurances sociales sont insuffisantes [2, 3].

- La discussion par le Comité central des résultats d'une consultation de la Société suisse pour la politique de la santé (cf. infra) à propos d'un système de compensation en cas de responsabilité sans faute [4].

- Une nouvelle discussion du Comité central sur le sujet, l'idée de la création d'une telle «assurance des patients» étant à nouveau soutenue [5], et un communiqué y relatif [6].

\section{Ce qui peut être fait}

Depuis des années, la Société suisse pour la politique de la santé (Schweizerische Gesellschaft für Gesundheitspolitik SGGP/SSPS) notamment insiste sur le besoin de créer un dispositif de compensation/ indemnisation pour les personnes victimes d'accidents médico-thérapeutiques. La collaboration de la Fédération des médecins suisses, des assureurs et de fondations privées a été recherchée; l'attention des partis politiques et d'autres instances a été sollicitée. La réalité oblige malheureusement à relever que ces démarches n'ont pas eu le succès voulu. Finalement, en 2000, l'actuel président de la société, le conseiller national Jost Gross, juriste thurgovien, a déposé une motion aux Chambres fédérales. ${ }^{2}$ Le Conseil fédéral a pris position le 27 novembre 2000, présentant les arguments qui à son avis ne permettent pas d'entrer en matière sur les différents modèles auxquels on peut penser et proposant d'écarter la motion.

Ainsi, malgré des positions de principe favorables dont celles de la FMH, le moins qu'on puisse dire est que l'accueil reçu a été tiède et les appuis concrets très insuffisants. Personne ou presque ne semble avoir estimé qu'il y avait là un objet suffisamment important pour qu'on y consacre du temps et des ressources.

Pourtant, il n'est pas difficile d'imaginer plusieurs dispositifs qui répondraient au moins dans une certaine mesure aux conséquences lourdes, parfois dra-

\footnotetext{
Des informations complémentaires et documents concernant cette motion et les efforts qui l'ont précédée peuvent être obtenus auprès de J. Gross, Dr. iur., et G. Kocher, Dr. rer. pol., Secrétariat de la SGGP/SSPS, Haldenweg 10A, 3074 Muri (e-mail: info@sggp.ch).
}

matiques, des aléas thérapeutiques. On peut envisager la forme d'une fondation privée (ce qu'ont cherché les efforts initiaux de la SGGP/SSPS) ou d'un système de droit public:

- création d'un fonds alimenté par une contribution régulière, très modeste, émanant des primes d'assurance-maladie ou accident de base (LAMal, LAA);

- une adjonction à la LAVI (loi fédérale sur l'aide aux victimes d'infractions) prévoyant un volet "accidents médicaux".

Il convient ici de redire que la Finlande, la Suède et la Nouvelle Zélande ont mis en place de tels systèmes, qui fonctionnent à satisfaction. On notera aussi que, dans un projet de loi présenté le 5 septembre 2001 et soumis à l'Assemblée nationale et au Sénat, le Gouvernement français propose la mise en place d'un mécanisme d'indemnisation des victimes d'accidents médicaux. La Société française de santé publique relève à ce sujet qu'il y a "urgence à légiférer". Dans un article récent, le Directeur de l'Institut de droit de la santé de l'Université de Neuchâtel concluait «le moment me paraît venu de réfléchir sérieusement, pragmatiquement et dans la perspective globale de la réparation du dommage corporel par les assurances sociales, à un système d'indemnisation des accidents médicaux" [7].

\section{Remarques de conclusion}

La présente contribution n'entend pas entrer dans le détail de ce qui pourrait être établi en Suisse, mais a pour but de vivement attirer l'attention de l'ensemble des partenaires concernés, y compris au niveau politique: dans un pays comme le nôtre, organisé, sérieux, disposant d'un bon système de santé (même si l'évolution et le financement de ce dernier posent plusieurs problèmes), il n'est plus vraiment admissible que soit maintenu un vide en ce qui concerne une compensation adéquate des conséquences d'aléas thérapeutiques. Et nous ne croyons pas qu'il soit judicieux d'attendre la réforme du droit de la responsabilité civile en général pour s'attaquer au problème.

Quelques mots encore pour évoquer des critiques qui peuvent être émises dans les discussions à ce sujet:

- Comme souvent, on décrira avec force les risques majeurs d'une "dérive à l'américaine». Mais on sait qu'une telle dérive (dans les dimensions qu'on connaît aux Etats-Unis) n'est simplement pas possible dans notre pays, à cause de différences majeures quant au cadre juridique et aux conditions d'exercice du droit.

- On allèguera aussi qu'un tel système va alimenter l'esprit revendicateur des malades, qui demanderont des compensations à tout propos et pour des bagatelles ... Une modalité concrète, parmi d'autres à préciser, qui peut prévenir ce risque (et une juridisation supplémentaire indésirable du système de santé) est de définir un plancher 
suffisamment élevé en dessous duquel on n'entre pas en matière sur une indemnisation. On peut imaginer que ce ne serait qu'à partir du moment où le patient ou ses proches présentent une argumentation crédible selon quoi l'aléa entraîne des conséquences financières dépassant Fr. 20000.-, par exemple, qu'on peut faire appel au fonds.

Il doit être clair que le dispositif a pour but essentiel de dédommager de manière acceptable ceux qui peuvent être appelés "accidentés de la médecine». C'est à l'évidence le cas de Cosette L. qui vient de retenir l'attention. Il y en a d'autres, qui méritent qu'on s'attache sans délai à trouver une solution adéquate à leur problème.

\section{Références}

1 Ott H. Responsabilité causale pour les médecins? Bull Méd Suisses 1991;72:1658-60 (deutsch: 802-4).

2 FMH. Délibérations du Comité central du 11 juillet 1991. Bull Méd Suisses 1991;72:1341.

3 FMH. Communiqué «Caisse des patients pour les cas de nécessité». Bull Méd Suisses 1991;72:1281.

4 FMH. Délibérations du Comité central du 15 décembre 1994. Bull Méd Suisses 1995;76:89.

5 FMH. Délibérations du Comité central du 3 février 1999. Bull Méd Suisses 1999;80:587.

6 FMH. Prise de position du Comité central. Indemnisation indépendante de la faute. Bull Méd Suisses 1999;80:777.

7 Guillod 0. Responsabilité médicale: La prise de la pastille. Médecine Et Hygiène 1999;57:886-9. 